\title{
Reproducible Documents with PythonTeX
}

\author{
Geoffrey M Poore \\ http: / / www - youtube. com/watch?v=G-UDHc2UVOg
}

\begin{abstract}
PythonTeX is a LaTeX package that allows Python code in a LaTeX document to be executed. This makes possible reproducible documents that combine analysis with the code required to perform it. Writing such documents can be more efficient because code is adjacent to its output. Writing is also less error-prone since results may be accessed directly from within the document, without copy-and-pasting. This paper provides an overview of PythonTeX, including Python output caching, dependency tracking, synchronization of errors and warnings with the LaTeX document, conversion of documents to other formats, and support for languages beyond Python. These features are illustrated through an extended, step-by-step example of reproducible analysis performed with PythonTeX.
\end{abstract}

Index Terms-reproducible science, reproducible documents, dynamic report generation

\section{Introduction}

The concept of "reproducible documents" is not new-indeed, there are at least two definitions, each with its own history.

According to one definition, a reproducible document is a document whose results may be conveniently reproduced via a makefile or a similar approach [Schwab]. Systems such as Madagascar [MAD] and VisTrails [VIS] represent a more recent and sophisticated version of this idea. The actual writing process for this type of document closely resembles the unreproducible case, except that the author must create the makefile (or equivalent), and thus it is easier to ensure that figures and other results are current.

According to another definition, a reproducible document is a document in which analysis code is embedded. The document itself both generates and reports results, using external data. This approach is common among users of the R language. Sweave has allowed R to be embedded in LaTeX since 2002 [Leisch]. The knitr package provides similar but more powerful functionality, and has become increasingly popular since its release in 2011 [Xie]. This approach to reproducible documents has roots in literate programming, through noweb [Ramsey] ultimately back to Knuth's original concept [Knuth]. Knuth suggested that programs be written as literature, interweaving code and documentation in a form geared toward human readers. Similarly, a reproducible document with embedded code integrates code and document into a unified whole. The writing process for such a document can be significantly different from the unreproducible case because of

* Corresponding author: gpoore@uu.edu

\$ Union University

Copyright $\odot 2013$ Geoffrey M Poore. This is an open-access article distributed under the terms of the Creative Commons Attribution License, which permits unrestricted use, distribution, and reproduction in any medium, provided the original author and source are credited. the tight integration that is possible. For example, it is possible to create dynamic reports with Sweave and knitr that automatically accommodate whatever data is provided.

These two definitions of a reproducible document need not be mutually exclusive. They might be thought of as two ends of a continuum, with a given project potentially benefiting from some combination. The makefile-style approach may be more appropriate for large codebases and complex computations, but even then, it can be convenient to embed plotting code in reports. Likewise, even a relatively simple analysis might benefit from externalizing some code and managing it via the makefile-style approach, rather than embedding everything.

This paper is primarily concerned with the second type of reproducible document, in which code is embedded. In the Python ecosystem, there are several options for creating such documents. The IPython notebook provides a highly interactive interface in which code, results, and text may be combined [IPY]. Reproducible documents may be created with Sphinx [Brandl], though the extent to which this is possible strongly depends on the extensions employed. Pweave is essentially Sweave for Python, with support for reST, Sphinx, and markdown in addition to LaTeX [Pastell]. There have also been LaTeX packages that allow Python code to be included in LaTeX documents: python. sty [Ehmsen], SageTeX [Drake], and SympyTeX [Molteno]. Python$\mathrm{TeX}$ is the most recent of these packages.

The LaTeX-based approach has some drawbacks. It is less interactive than the IPython notebook. And it can be less convenient than a non-LaTeX system for converting documents to formats such as HTML. At the same time, a LaTeX package has several significant advantages. Since the user directly creates a valid LaTeX document, the full power of LaTeX is immediately accessible. A LaTeX package can also provide superior LaTeX integration compared to other approaches that do support LaTeX but are not integrated at the package level. For example, PythonTeX makes it possible to create LaTeX macros that contain Python code.

The PythonTeX package builds on previous LaTeX packages, emphasizing performance and usability. Python code may be divided into user-defined sessions, which automatically run in parallel via the multiprocessing module [MULT]. All code output is cached and the user has fine-grained control over when code will be re-executed, including the option to track document dependencies. This allows a PythonTeX document to be compiled just as quickly as a normal LaTeX document so long as no Python code is modified. Python errors and warnings are synchronized with the document's line numbering, so that their source is easily located. PythonTeX documents may be easily converted to plain LaTeX documents suitable for journal submission or format 
conversion. While PythonTeX's focus is on Python, the package may extended to support additional languages.

\section{PythonTeX Overview}

Using the PythonTeX package is as simple as adding the command

\usepackage $\{$ pythontex

to the preamble of a LaTeX document and slightly modifying the way you compile the document. When a document using the PythonTeX package is first compiled, all of the Python code contained in the document is saved to an auxiliary file (with delimiters). To execute the Python code, you simply run the provided script pythontex.py with the document name as an argument. In a standard PythonTeX installation, a symlink or launching wrapper for this script is created in your TeX installation's bin/ directory, so that the script will be on your PATH. The next time you compile the document, all Python-generated content will be included.

PythonTeX is compatible with all standard LaTeX engines (executable binaries): pdfTeX, XeTeX, and LuaTeX. It has been tested with TeX Live [TL] and MiKTeX [MIK], and should work with other distributions.

\section{Commands and Environments}

PythonTeX provides a number of LaTeX commands and environments. These can be used to run any valid Python code; even imports from __future__ are allowed, so long as they occur before any other code.

The code environment runs whatever code is provided. By default, any printed content is automatically included in the document. For example,

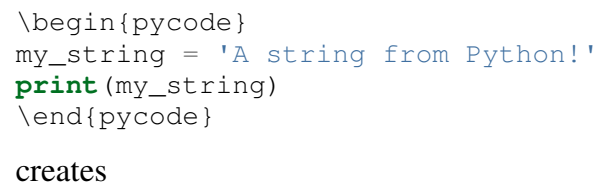

creates

\section{A string from Python!}

The block environment also executes its contents. In this case, the code is typeset with highlighting from Pygments [PYG]. Printed content is not automatically included, but may be brought in via the $\backslash$ printpythontex command. For example,

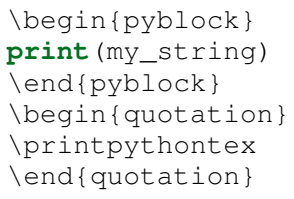

typesets

print (my_string)

\section{A string from Python!}

All commands and environments take an optional argument that specifies the session in which the code is executed. If a session is not specified, code is executed in a default session. In the case above, the variable my_string was available to be printed in the block environment because the block environment shares the same default session as the code environment.

Inline versions of the code and block environments are provided as the commands $\backslash$ pyc and $\backslash$ pyb. A special command $\backslash p y$ is provided that returns a string representation of its argument. For example, \py $\{2 \star \star 8\}$ yields 256 .

PythonTeX also provides a verbatim command $\backslash$ pyv and environment pyverbatim. These simply typeset highlighted code; nothing is executed. Descriptions of additional commands and environments are available in the documentation.

\section{Caching}

All Python output is cached. PythonTeX also tracks the exit status of each session, including the number of errors and warnings produced (it parses stderr). By default, code is only re-executed by pythontex.py when it has been modified or when it produced errors on the last run.

That approach is most efficient for many cases, but sometimes the user may need finer-grained control over code execution. This is provided via the package option rerun, which accepts five values:

- never: Code is never executed; only syntax highlighting is performed.

- modified: Only modified code is executed.

- errors: Only modified code or code that produced errors on the last run is executed.

- warnings: Code is executed if it was modified or if it produced errors or warnings previously.

- always: Code is always executed.

\section{Tracking Dependencies and Created Files}

Code may need to be re-executed not just based on its own modification or exit status, but also based on external dependencies.

PythonTeX includes a Python class that provides several important utilities. An instance of this class called pytex is automatically created in each session. The utilities class provides an add_dependencies () method that allows dependencies to be specified and tracked. Whenever PythonTeX runs, all dependencies are checked for modification, and all code with changed dependencies is re-executed (unless rerun=never). By default, modification is detected via modification time (os.path.getmtime () ) [OSPATH], since this is fast even for large data sets. File hashing may be used instead via the package option hashdependencies.

The PythonTeX utilities class also provides an add_created () method. This allows created files to be deleted automatically when the code that created them is re-executed, preventing unused files from accumulating. For example, if a figure is saved under one name, and later the name is changed, the old version would be deleted automatically if it were tracked.

When there are only a few dependencies or created files, it may be simplest to specify them manually. For example, the line

pytex.add_dependencies ('data.txt')

could be added after data.txt is loaded. In cases where the manual approach is tedious, the entire tracking process may be automated. A custom version of open () could be defined in which each file opened is tracked based on whether it is opened for reading (dependency) or writing (created).

\section{Synchronizing Errors and Warnings}

When pythontex.py runs, it prints an annotated version of the stderr produced by user code. Before each error or warning, a 
message is inserted that specifies the corresponding line number in the document. For example, if the code environment

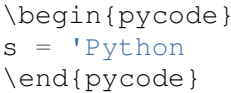

were on line 20 of a document, then when PythonTeX runs, it would return a message in the form

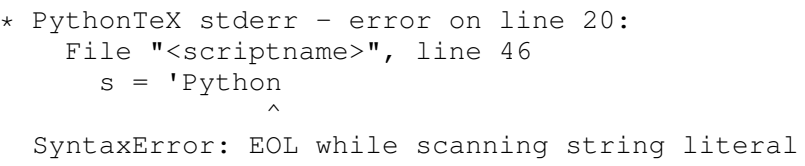

where <scriptname $>$ is the name of the temporary script that was executed. This greatly simplifies debugging.

PythonTeX provides a sophisticated system that parses stderr and synchronizes line numbers in errors and warnings with the document's line numbering. As PythonTeX assembles the code to be executed, it creates a record of where each chunk of code originated in the document. The actual scripts that are executed are assembled by inserting user code into predefined templates that provide access to the PythonTeX utilities class and additional functionality. This means that the line numbers of the code that is actually executed differ not only from the document's line numbering, but also from the user code's numbering. In the example above, the error occurred on line 20 of the document, on line 46 of the code that was actually executed, and on line 1 of the user code. PythonTeX keeps a running tally of how many lines originated in user code versus templates, so that the correct line number in the document may be calculated.

In some cases, errors or warnings may only reference a line number in the file in which they occur. For example, if warnings.warn() [WAR] is used in an imported module, a line number in the module will be referenced, but a line number in the code that imported the module will not. The previous approach to synchronization fails. To deal with this scenario, PythonTeX writes delimiters to stderr before each command and environment. This allows messages that do not reference a line number in the user's code to be tracked back to a single command or environment in the document.

\section{Converting PythonTeX Documents}

One disadvantage of a reproducible document created with PythonTeX is that it mixes plain LaTeX with Python code. Many publishers will not accept documents that require specialized packages. In addition, some format converters for LaTeX documents only support a subset of LaTeX commands-so PythonTeX support is not an option.

To address these issues, PythonTeX includes a depythontex utility. It creates a version of a document in which all Python code has been replaced by its output. There is no way to tell that the converted document ever used PythonTeX. Typically, the converted document is a perfect copy of the original, though occasionally spacing may be slightly different based on the user's choice of depythontex options. A few features are especially noteworthy.

- Any Python-generated figures that were included in the original document will be included in the converted document; the converted document still checks the same paths for figures. It is possible to configure PythonTeX so that figures created by matplotlib [MPL] and other plotting libraries are automatically included in the document, without the user needing to enter an \includegraphics command. (Additional details are provided in the documentation.) Even in these cases, figures are correctly included in the converted document.

- Any code highlighted by PythonTeX in the original version can also be highlighted in the depythontex version. Highlighted code can be converted into the format of the listings [LST], minted [MINT], or fancyvrb [FV] packages for LaTeX. Line numbering and syntax highlighting are preserved if the target package supports them.

\section{When Python Is Not Enough}

While PythonTeX is focused on providing Python-LaTeX integration, most of the LaTeX interface is language-agnostic. In many cases, adding support for an additional language is as simple as providing two templates and creating a new instance of a Python class that defines languages. For example, support for Ruby has just been added to PythonTeX. This required two Ruby templates and a few lines of Python-only about 70 lines of code total. Most of the Ruby code simply implements a Ruby version of the PythonTeX utilities class, which manages dependencies, created files, and LaTeX integration. Part of this process also involved specifying the format of Ruby errors, warnings, and associated line numbers, so that Ruby errors and warnings can be synchronized with the document.

Support for additional languages will be added in the near future.

\section{Case Study: Average Temperatures in Austin, TX}

The remainder of this paper illustrates the application of PythonTeX through a reproducible analysis of average temperatures in Austin, TX. I will calculate monthly average high temperatures in 2012 at the Austin-Bergstrom International Airport from daily highs. In addition to demonstrating the basic features of PythonTeX, this example shows how performance may be optimized and how the final document may be converted to other formats.

\section{Data Set}

Daily high temperatures for 2012 at the Austin-Bergstrom International Airport were downloaded from the National Oceanic and Atmospheric Administration (NOAA)'s National Climatic Data Center [NCDC]. The data center's website provides a data search page. Setting the zip code to 78719 and selecting "Daily CHCND" accesses daily data at the airport. Maximum temperature TMAX was selected under the "Air temperature" category of daily data, and the data were downloaded in comma-separated values (CSV) format. The CSV file contained three columns: station name (the airport station's code), date (ISO 8601), and TMAX (temperature in tenths of a degree Celsius). The first three lines of the file are shown below:

STATION, DATE, TMAX GHCND : USW0 $0013904,20120101,172$ GHCND : USW0 $0013904,20120102,156$

Since the temperatures are in tenths of a degree Celsius, the 172 in the second line is 17.2 degrees Celsius. 


\section{Document Setup}

I will use the same IEEEtran document class used by the SciPy proceedings, with a minimal preamble. All Python sessions involved in the analysis should have access to the pickle module [PKL] and to lists of the names of the months. PythonTeX provides a pythontexcustomcode environment that is used to add code to all sessions of a given type. I use that environment to add the pickle import and the lists to all sessions for the py family of commands and environments (pycode, pyblock, $\backslash$ pyc, \pyb, \py, etc.).

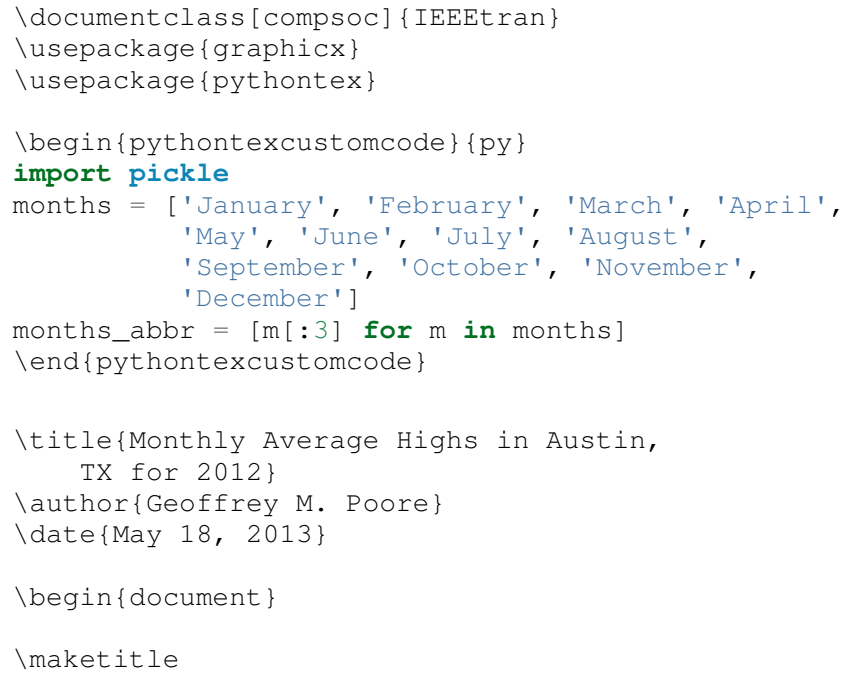

\section{Loading Data and Tracking Dependencies}

The first step in the analysis is loading the data. Since the data set is relatively small (daily values for one year) and in a simple format (CSV), it may be completely loaded into memory with the built-in open ( ) function.

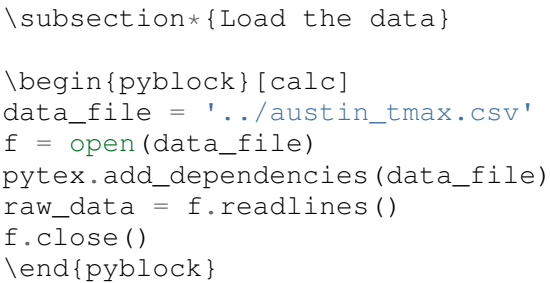

Notice the optional argument calc for the pyblock environment. I am creating a session $\mathrm{calc}$ in which I will calculate the monthly average highs. Later, I will save the final results of the calculations, so that they will be available to other sessions for plotting and further analysis. In this simple example, dividing the tasks among multiple sessions provides little if any performance benefit. But if I were working with a larger data set and/or more intensive calculations, it could be very useful to separate such calculations from the plotting and final analysis. That way, the calculations will only be performed when the data set or calculation code is modified.

The data file austin_tmax.CSV is located in my document's root directory. Since the PythonTeX working directory is by default a PythonTeX directory created within the document directory, I have to specify a relative path to the data file. I could have set the working directory to be the document directory instead, via \setpythontexworkingdir $\{$. . But this way all saved files will be isolated in the PythonTeX directory unless a path is specified, keeping the document directory cleaner.
The data file austin_tmax.Csv is now a dependency of the analysis. The analysis should be rerun in the event the data file is modified, for example, if a better data set is obtained. Since this is a relatively simple example, I add the dependency manually via add_dependencies (), rather than creating a custom version of open () that tracks dependencies and created files automatically.

\section{Data Processing}

Now that the data are loaded, they may be processed. The first row of data is a header, so it is ignored. The temperature readings are sorted into lists by month. Temperatures are converted from tenths of a degree Celsius to degrees Celsius. Finally, the averages are calculated and saved. The processed data file is added to the list of created files that are tracked, so that it is deleted whenever the code is run again. This ensures that renaming the file wouldn't leave old versions that could cause confusion.

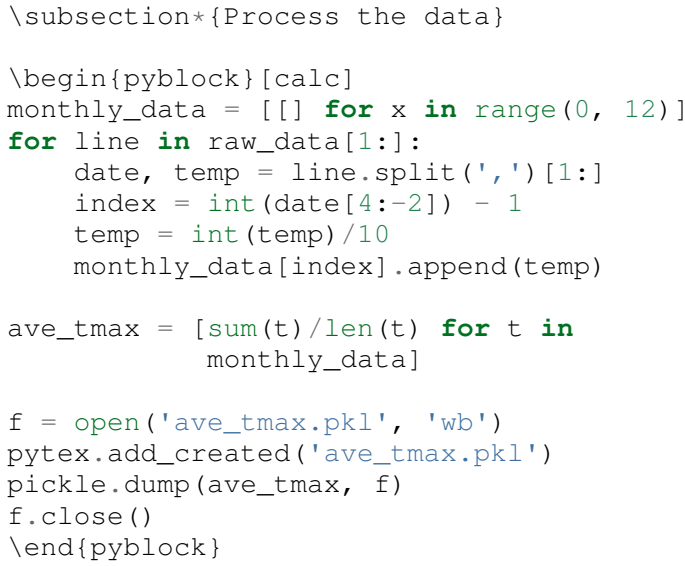

\section{Plotting}

Once the calculations are finished, it is time to plot the results. This is performed in a new session. Notice that pickle and the list of months are already available since they were added to all sessions via pythontexcust omcode. As before, dependencies and created files are specified. In this particular case, I have also matched the fonts in the plot to the document's fonts.

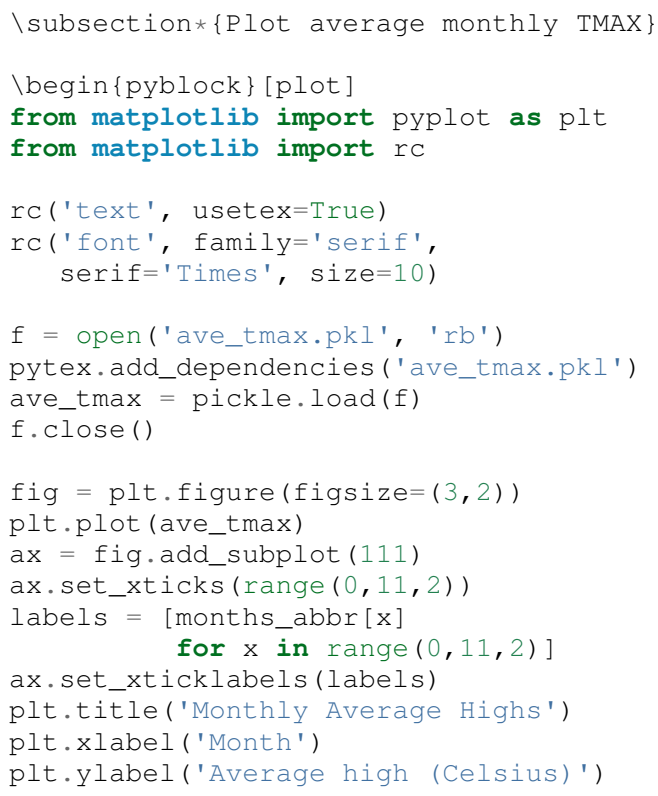


plt.xlim(0, 11)

plt.ylim(16, 39)

plt.savefig('ave_tmax.pdf', bbox_inches='tight' )

pytex.add_created ('ave_tmax.pdf')

lend $\{$ pyblock $\}$

$\backslash$ includegraphics [width=3in] \{ave_tmax.pdf $\}$

\section{Summary of Results}

It might be nice to add a summary of the results. In this case, I simply add a sentence giving the maximum monthly average temperature and the month in which it occurred. Notice the way in which Python content is interwoven with the text. If a data set for a different year were used, the sentence would update automatically.

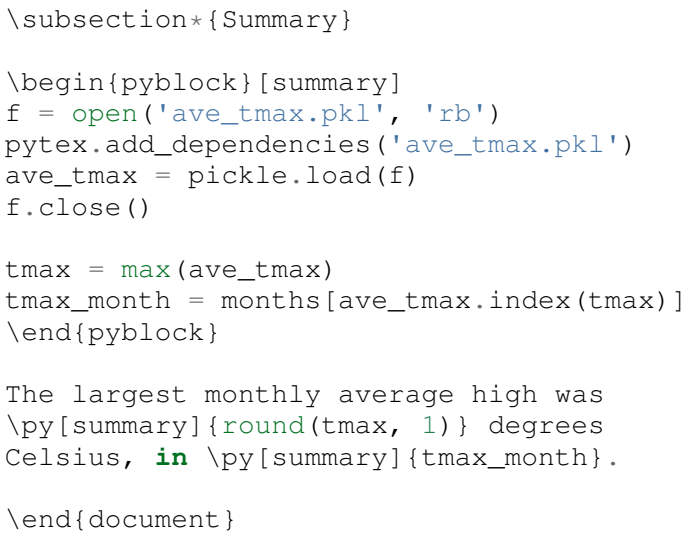

\section{Output and Conversion}

I compile the document to PDF by running pdflatex, then pythontex.py, and finally pdflatex on the file. The output is shown in Figure 1.

To compile this particular document, I have to run pythontex.py twice in a row. The first run creates the saved data in ave_tmax.pkl. The second run gives the plot and summary sessions access to the saved data. Since all sessions are executed in parallel, there is no guarantee that the data file will be created before the plot and summary sessions try to access it. If the data file does not exist, these sessions produce errors during the first run and are automatically re-executed during the second run.

The analysis is complete at this point if a PDF is all that is desired. But perhaps the analysis should also be posted online in HTML format. A number of LaTeX-to-HTML converters exist, including TeX4ht [TEX4HT], HEVEA [HEVEA], and Pandoc [PAN]. I will use Pandoc in this example since the document has a simple structure that Pandoc fully supports. A different converter might be more appropriate for a more complex document.

Since Pandoc only supports a basic subset of LaTeX, it is not aware of the PythonTeX commands and environments and cannot convert the document in its current form. This is where the depythontex utility is needed. To use depythontex, I modify the case study document by adding the depythontex option when the PythonTeX package is loaded:

Iusepackage [depythontex] \{pythontex

I also edit the document so that the figure is saved as a PNG rather than a PDF, so that it may be included in a webpage. Next, I compile the document with LaTeX, run the PythonTeX script, and compile again. This creates an auxiliary file that depythontex needs. Then I run depythontex on the case study document:

depythontex casestudy.tex --listing=minted

This creates a file depythontex_casestudy.tex in which all PythonTeX commands and environments have been replaced by their output. The depythontex utility provides a --listing option that determines how PythonTeX code listings are translated. In this case, I am having them translated into the syntax of the minted package [MINT], since Pandoc can interpret minted syntax. Next, I run Pandoc on the depythontex output:

pandoc --standalone depythontex_casestudy.tex - o casestudy.html

Together, casestudy.html and ave_tmax.png provide an HTML version of casestudy . tex, including syntax highlighting (Figure 2).

\section{Conclusion}

PythonTeX provides an efficient, user-friendly system for creating reproducible documents with Python and LaTeX. Since code output is cached and user-defined sessions run in parallel, document compile times are minimized. Errors and warnings are synchronized with the document's line numbering so that debugging is simple. Because PythonTeX documents can be converted to plain LaTeX documents, the system is suitable for writing journal papers and documents that must be converted to other formats.

Most of the key elements planned for PythonTeX are already in place, but several significant enhancements are coming in the future. Support for additional languages will be added soon. Better support for macro programming with PythonTeX that mixes Python and LaTeX code is also under development. Several usability enhancements are in preparation, including the option to automatically include stderr in the document, next to its source, as an aid in debugging.

PythonTeX is under active development and provides many features not discussed here. Additional information and the latest release are available at https://github.com/gpoore/pythontex.

\section{REFERENCES}

[Schwab] M. Schwab, M. Karrenbach, and J. Claerbout. Making scientific computations reproducible. Computing in Science \& Engineering, 2(6):61-67, Nov/Dec 2000.

[MAD] Madagascar. http://www.ahay.org/.

[VIS] VisTrails. http://www.vistrails.org/.

[Leisch] F. Leisch. Sweave: Dynamic generation of statistical reports using literate data analysis, in Wolfgang Härdle and Bernd Rönz, editors, Compstat 2002 - Proceedings in Computational Statistics, pages 575580. Physica Verlag, Heidelberg, 2002. ISBN 3-7908-1517-9. http: //www.statistik.lmu.de/ leisch/Sweave/.

[Xie] Y. Xie. "knitr: Elegant, flexible and fast dynamic report generation with R." http://yihui.name/knitr/.

[Ramsey] N. Ramsey. Literate programming simplified. IEEE Software, 11(5):97-105, September 1994. http://www.cs.tufts.edu/ nr/noweb/.

[Knuth] D. E. Knuth. Literate Programming. CSLI Lecture Notes, no. 27. Stanford, California: Center for the Study of Language and Information, 1992.

[Brandl] G. Brandl. "SPHINX: Python Documentation Generator." http:// sphinx-doc.org/.

[Pastell] M. Pastell. "Pweave - reports from data with Python." http://mpastell. com/pweave/.

[IPY] The IPython development team. "The IPython Notebook." http: //ipython.org/notebook.html.

[Ehmsen] M. R. Ehmsen. "Python in LaTeX." http://www.ctan.org/pkg/ python. 


\title{
Monthly Average Highs in Austin, TX for 2012
}

\author{
Geoffrey M. Poore
}

\author{
Load the data \\ data_file $=$ '. ./austin_tmax.csv' \\ $\mathrm{f}=$ open (data_file) \\ pytex.add_dependencies (data_file) \\ raw_data = f.readlines () \\ f.close ()
}

\section{Process the data}

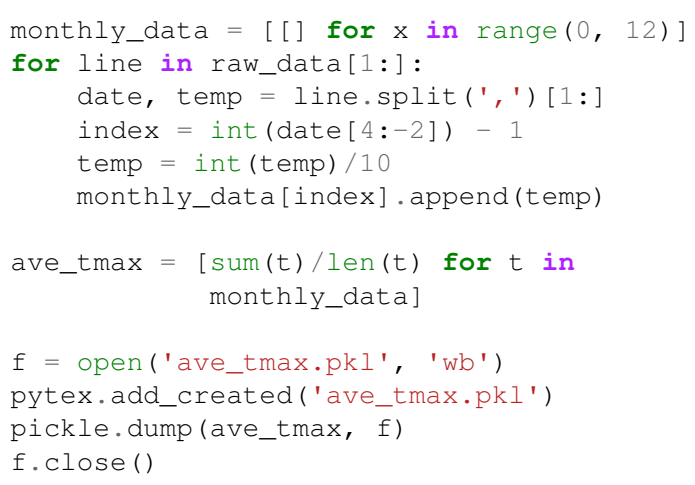

\section{Plot average monthly TMAX}

from matplotlib import pyplot as plt from matplotlib import $\mathrm{rC}$

rc ('text', usetex=True)
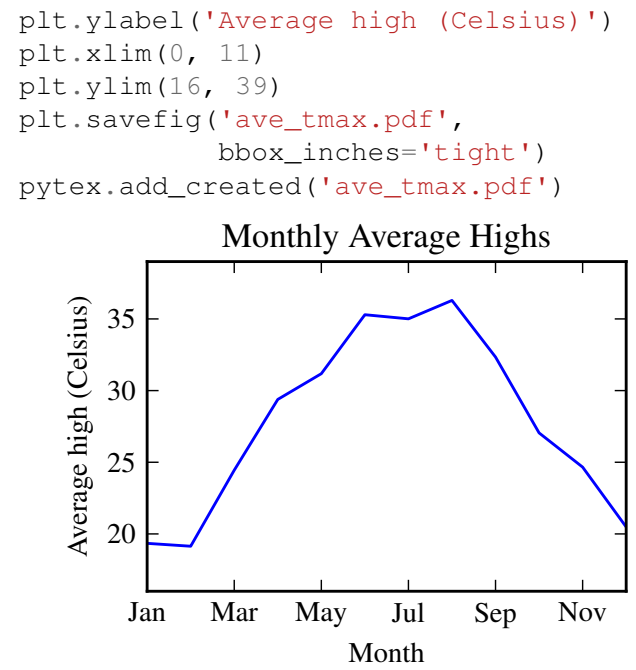

\section{Summary}

$f=$ open ('ave_tmax.pkl', 'rb')

pytex.add_dependencies ('ave_tmax.pkl')

ave_tmax $=$ pickle.load $(\mathrm{f})$

f.close ()

$t \max =\max \left(a v e \_t \max \right)$

tmax_month = months [ave_tmax.index (tmax)]

The largest monthly average high was 36.3 degrees Celsius, in August.

Fig. 1: The PDF version of the temperature case study. 


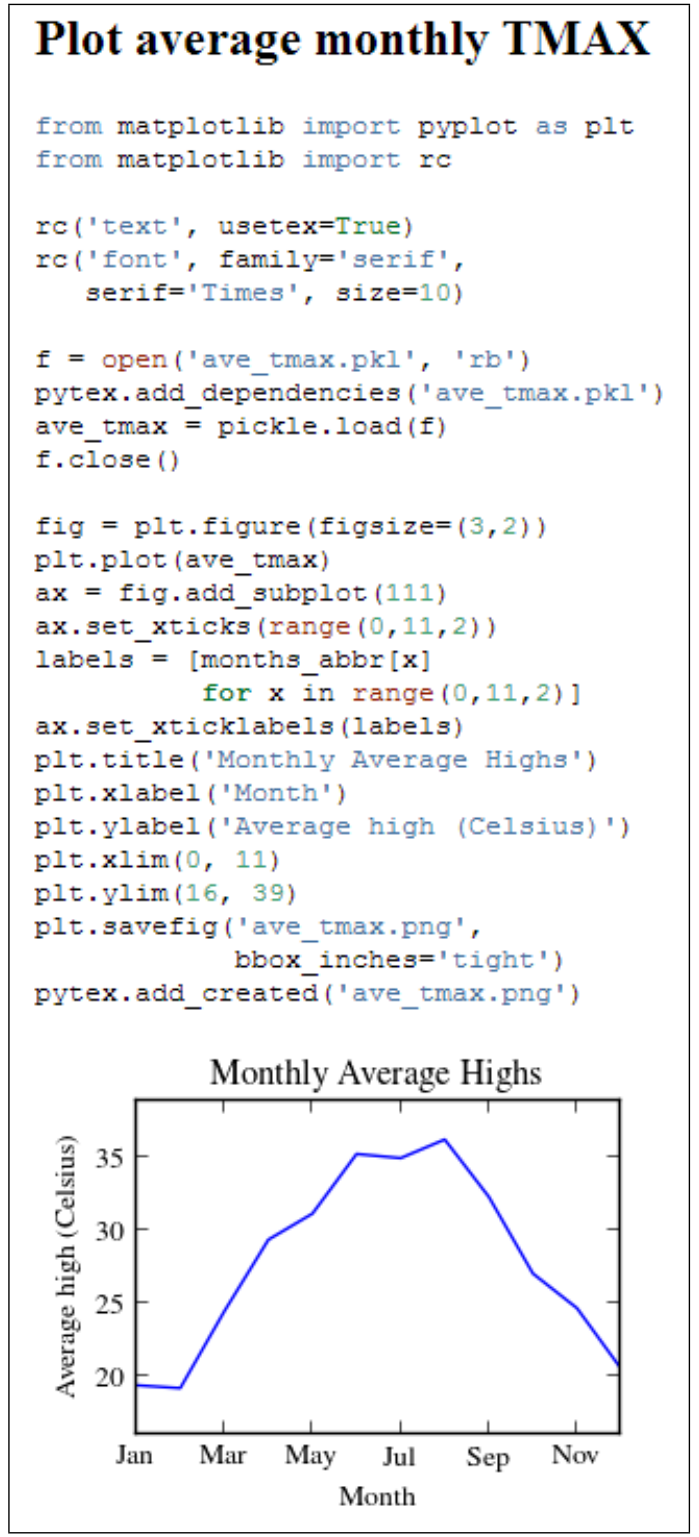

Fig. 2: A screenshot of part of the HTML version of the case study.

[Drake] D. Drake. "The SageTeX package." https://bitbucket.org/ddrake/ sagetex/.

[Molteno] T. Molteno. "The sympytex package." https://github.com/tmolteno/ SympyTeX/.

[MULT] Python Software Foundation. "multiprocessing - Processbased 'threading' interface." http://docs.python.org/2/library/ multiprocessing.html.

[TL] TeX Live. http://www.tug.org/texlive/.

[MIK] MiKTeX. http://www.miktex.org/.

[WAR] Python Software Foundation. "warnings - Warning control." http://docs.python.org/2/library/warnings.html.

[PYG] The Pocoo Team. "Pygments: Python Syntax Highlighter." http:// pygments.org/.

[MPL] J. D. Hunter. Matplotlib: A 2D Graphics Environment, in Computing in Science \& Engineering, Vol. 9, No. 3. (2007), pp. 90-95. http: //matplotlib.org/.

[LST] C. Heinz and B. Moses. "The Listings Package." http://www.ctan. org/tex-archive/macros/latex/contrib/listings/.

[FV] T. Van Zandt, D. Girou, S. Rahtz, and H. Voß. "The 'fancyvrb' package: Fancy Verbatims in LaTeX." http://www.ctan.org/pkg/fancyvrb.

[NCDC] National Climatic Data Center. http://www.ncdc.noaa.gov.

[PKL] Python Software Foundation. "pickle - Python object serialization." http://docs.python.org/2/library/pickle.html.
[OSPATH] Python Software Foundation. "os.path - Common pathname manipulations." http://docs.python.org/2/library/os.path.html.

[TEX4HT] TeX User's Group. http://www.tug.org/applications/tex4ht/.

[HEVEA] L. Maranget. "HEVEA." http://hevea.inria.fr/.

[PAN] J. MacFarlane. "Pandoc: a universal document converter." http: //johnmacfarlane.net/pandoc/.

[MINT] K. Rudolph. "The minted package: Highlighted source code in LaTeX." https://code.google.com/p/minted/. 